\title{
Chapter 10 The roles of GEN and CON in modeling ternary rhythm
}

\author{
Curt Rice
}

\section{Introduction}

Structures absent from the set of well-formed representations in natural language can be avoided by restricting the alphabet or the operations which are inherently available in Universal Grammar. Alternatively, they can be suppressed by restrictions articulated in every individual grammar. In Optimality Theory, the first of these strategies would be modeled through restrictions on GEN, and the second via the interaction of constraints in CON (Prince and Smolensky 1993). ${ }^{1}$ The present article aims to elucidate these options by considering competing strategies for approaching metrical analyses of ternary rhythm in OT.

I begin by setting the theoretical stage for further exploration of a division of labor between GEN and CON, including a selective review of the guidance offered in the literature on this matter. This is followed by a reminder of the analytical challenges which ternary rhythm presents as we review the two dominant strategies found in metrical theory for modeling such patterns. The remainder of the paper is devoted to schematic discussion of OT strategies and their implications for GEN and CON as potential subdivisions in which restrictions on structure might reside.

The contributions of the paper are twofold. Specific problems are identified where a solution could either be in GEN or CON, and the relative merits of those possible solutions are discussed. In this way, I aspire to stimulate more explicit engagement with the properties of GEN including specific restrictions on freedom of analysis. Secondarily, the paper makes clear that we currently lack an OT strategy for modeling ternary rhythm which achieves its results exclusively as an emergent effect from constraint interaction. Instead, I demonstrate that even the best effort has explicit ternary-specific stipulations lurking in the shadows. While my intention here is not to provide an analysis 


\section{Curt Rice}

of ternary rhythm - which is introduced for the present purposes to occasion a discussion of the division of labor between GEN and CON - a set of issues are nonetheless identified which future work on ternary rhythm must consider.

\section{Phonological structure in OT}

The advent of Optimality Theory correlates chronologically with the near cessation of work on the structure of phonological entities. The severed lines of research include many of those most prominent in the 1980s, such as feature geometry, the internal structure of the syllable and the metrical theory of stress, to name just a few.

Part of the responsibility for this state of affairs surely resides in the fact that OT is itself not a theory of structure. Indeed, no theory of the structure of any grammatical entity is inherently in conflict with an OT approach to modeling grammar; arguments advancing one theory of structure against another therefore have no bearing on the validity of OT. With OT, the focus of research shifts to the properties of constraints and their interaction and to other aspects of the theory, in part with the goal of determining the potential of a parallel model vis-à-vis derivational approaches.

There is a second culprit bearing responsibility for the reduction in work on phonological structure, namely the GEN-defining principle of freedom of analysis which simply states that 'any amount of structure may be posited' McCarthy and Prince (1993: 21). ${ }^{2}$ GEN receives an input, and freedom of analysis operates to posit structure, spouting candidates which may be identical to the input, but which may also have either less or more structure than the input.

[Gen] generates for any given input a large space of candidate analyses by freely exercising the basic structural resources of the representational theory. The idea is that the desired output lies somewhere in this space, and the constraint system of the grammar is strong enough to single it out.

(Prince and Smolensky 1993: 6)

One nearly reads the reference to 'basic structural resources' as an invitation to pursue research on the nature of said resources, but acceptance is hindered by frequent assertion of the proliferation of unrestricted structure.

The GEN function for syllable structure should admit every conceivable structure, with every conceivable array of affiliations and empty and filled nodes.

(Prince and Smolensky 1993: 26) 
We see with these quotes that GEN with its principle of freedom of analysis is presented in classical OT as producing any structure, thereby undermining the need for research to identify which particular structures should be available for manipulation. The division of labor between GEN and CON in the foundational OT literature is clear: GEN can do anything, we needn't say more; for results, look to CON.

And although the door to research on basic structural resources is at times cracked open to rule out definitionally ill-formed structures - syllables dominating feet, crossing association lines, perhaps nonextant distinctive features - the opening just isn't quite wide enough to step through with a serious scientific agenda in hand.

Since GEN is the same in every language, it initially seems like a good place to deposit a wide variety of "hard" universals [...] There is a flaw here, though. Hardwiring universals into GEN is inevitably a matter of brute-force stipulation, with no hope of explanation or connection to other matters - it is the end of discussion rather than the beginning.

(McCarthy 2002: 8-9).

Hardwiring may indeed be the end of a discussion. But that need not make the discussion itself any less interesting or important, nor does it make the conclusion incorrect. Furthermore, it is not a priori inconceivable that hardwired universals can be connected 'to other matters'. Finally, in the context of this quote, the student of OT might be forgiven for thinking that at least in practice, solutions derived from $\mathrm{CON}-$ e.g. through the positing of new constraints - aren't immune from the charge of being 'brute-force' either.

Solutions in CON are successful when typological effects emerge from the interaction of independently motivated constraints. Effects which do not emerge in this way might legitimately find their explanation in the structure of GEN. To pursue a discussion of the roles of these two potential solution loci, we turn now to the matter of ternary rhythm.

\section{Ternary rhythm in metrical theory}

Iterative ternary rhythm in natural language is well-documented, as is the lack of consensus about the implications of these patterns for foot typology. ${ }^{3}$ Of particular relevance for us is the disagreement about whether the existence of iterative ternary rhythm compels positing ternary metrical feet. If it does, 


\section{Curt Rice}

what are the properties of these feet, e.g. can the head be anywhere in the foot, do the feet have internal structure? Should those properties follow from GEN or CON? If ternary rhythm does not compel ternary feet, should they be ruled out in UG by a restriction on freedom of analysis? Should they be banned in CON by a single constraint or does their absence follow from the interaction of several constraints? In short, what are the contributions of GEN to the analysis of ternary rhythm? What are the contributions of CON and Eval?

Consider first some patterns and the initial reactions to these patterns in the metrical phonology literature. The Bolivian language Cayuvava shows a ternary alternation which can be schematized as in (1), where stress is on every third syllable counting from the right edge of the word Key (1961, 1967). Each number represents a syllable; 0 represents no stress; 1 represents primary stress; 2 represents secondary stress. The words are right-justified because the pattern is claimed to emanate from the right edge of the word. Words of such length are provided in the source materials.

In her analysis of Cayuvava, Levin (1988) relaxes metrical theory to allow for amphibrachs, i.e. ternary feet with prominence on the middle syllable. To give Cayuvava an amphibrachic parse correctly locating stress, Levin uses final extrametricality, which can be overridden when necessary to build at least one foot on the (minimal) disyllabic words. In longer words, initial lone syllables are left unfooted. In (1), parentheses indicate feet and angled brackets mark extrametricality.

$$
\begin{array}{cr}
\multicolumn{2}{c}{\text { Ternary }} \\
\text { a. } & (10) \\
\text { b. } & (10)\langle 0\rangle \\
\text { c. } & (010)\langle 0\rangle \\
\text { d. } & 0(010)\langle 0\rangle \\
\text { e. } & (20)(010)\langle 0\rangle \\
\text { f. } & (020)(010)\langle 0\rangle \\
\text { g. } & 0(020)(010)\langle 0\rangle \\
\text { h. } & (20)(020)(010)\langle 0\rangle \\
\text { i } & (020)(020)(010)\langle 0\rangle
\end{array}
$$

Halle and Vergnaud (1987) pursue Levin's strategy and limit UG to this one type of ternary foot, arguing that there is no compelling case of iterative dactyls or anapests. Their strategy is to parameterize a head terminal re- 
quirement, with amphibrachs having the negative value. A [-head terminal] constituent is one which allows the head to be separated from the edge of the constituent by maximally one syllable (or mora), generating the possibility of iterative amphibrachs, cf. Rice (1988) for related discussion. The ternary foot approach receives further elaboration and restriction in the metrical theory literature, including an approach with internal structure, advocated in works such as Dresher and Lahiri (1991) and Rice (1992); see also related discussion in van der Hulst (1999).

The alternative to an analysis with ternary feet begins with the assumption that UG is limited to the foot inventory in (2) (Hayes 1995).

$\begin{array}{lcccc}\text { The Hayesian foot typology } & & \\ \text { a. Syllabic trochee } & (\mathrm{x} & .) & & \\ & \sigma & \sigma & & \\ & & & & \\ \text { b. Moraic trochee } & (\mathrm{x} & .) & & (\mathrm{x}) \\ & \mathrm{L} & \mathrm{L} & \text { or } & \mathrm{H} \\ & & & & \\ \text { c. Iamb } & (. & \mathrm{x}) & & (\mathrm{x}) \\ & \mathrm{L} & \sigma & \text { or } & \mathrm{H}\end{array}$

Ternary rhythm, of course, cannot be modeled with the exhaustive parsing of a string using any of these feet. But it can be modeled with non-exhaustive parsing, and that is the strategy which Hayes advocates. Instead of being endowed with a particular type of ternary foot, UG is endowed with a parameter which requires with its so-called weak local parsing setting that feet be constructed iteratively but non-exhaustively, with an unparsed syllable between each foot. By limiting the intervening material to maximally one (light) syllable, there can be maximally two unstressed syllables between stressed ones, giving a ternary pattern - but not more - using only binary feet.

For a ternary pattern such as that seen in Cayuvava, trochees are constructed from right to left, with final extrametricality and with weak local parsing. In (3c), the final syllable is unfooted by extrametricality and the initial syllable is unfooted because it is too little to be a foot. In (d) the powerful effects of weak local parsing are seen; here there is in fact sufficient material to form a foot, but doing so would result in adjacent feet, which is not allowed by the parsing strategy. Not until we have six syllables, as in (e), 
(3) Ternary alternations parsed into nonexhaustive binary feet

a.

b.

c.

d.

e. $\quad(20) 0(10)\langle 0\rangle$

f. $\quad 0(20) 0(10)\langle 0\rangle$

g. $\quad 00(20) 0(10)\langle 0\rangle$

h. $(20) 0(20) 0(10)\langle 0\rangle$

i $0(20) 0(20) 0(10)\langle 0\rangle$

is there sufficient space to build two non-adjacent - i.e. weakly local parsed - feet.

\section{Amphibrachs in OT}

I begin an investigation of the roles of GEN and CON in modeling ternary rhythm by exploring an OT analysis using amphibrachs. To accomplish this, freedom of analysis must obviously be allowed to construct candidates with ternary feet. The discussion here will contribute to our larger goal of examining the trade-off between GEN and CON through a consideration of the status of degenerate feet in a ternary system. Because I am not presenting a thorough analysis of ternary stress, but am rather using ternary stress as an occasion to discuss the architecture of OT, I will make some assumptions which must ultimately be justified in a theory of ternary rhythm. For example, I will follow Halle and Vergnaud (1987) in assuming the absence of dactyls and anapests, and will say nothing more about this here. I will also leave aside any discussion of feet larger than ternary ones.

\subsection{Optimizing amphibrachs}

To optimize a parse with amphibrachs, CON must be augmented with a constraint which can dominate the well established FOOTBINARITY constraint that requires a foot to be parsable as binary at the level of either the syllable or the mora (Prince and Smolensky 1993). One strategy for optimizing amphibrachs would be a constraint which explicitly rewards parses with this foot type. Prince and Smolensky achieve footing with the constraint RHTYPE=I/T 
which they describe as 'a constraint which sets the rhythmic type at either iambic or trochaic' with clear reference to the Hayesian typology (Prince and Smolensky 1993: 56). Amphibrachs could be optimized if this constraint were provided with a third setting, RHTYPE $=\mathrm{I} / \mathrm{T} / \mathrm{A}$, allowing the specification of an amphibrach, which would be violated by any foot not having a series of three syllables with stress on the middle one. Such an approach would correspond to expanding Hayes' typology in (2) to include the amphibrach, a foot which has a flat ternary structure dominating three light syllables or three morae, the middle of which bears stress.

The effect of final extrametricality can be achieved with high ranking NONFINALITY, which prohibits the head foot of the prosodic word from being word-final (Prince and Smolensky1993: 45), along with ALIGnR(WD, $\mathrm{H}_{P r W d}$ ), which requires the same foot to be aligned with the right edge of the word (McCarthy and Prince 1993: 34). When NONFINALITY is highest, these two constraints will place the first amphibrach as in the parses in (1). In the tableaux below, these two constraints are assumed in the suggested ranking and we therefore consider only candidates which satisfy NonFinALITY and which violate ALIGNR(WD, $\mathrm{H}_{P r W d}$ ) exactly once, i.e. candidates which have one 'extrametrical' syllable.

\subsection{Degenerate ternary feet}

The challenging patterns in (1) are those with imperfect parses, such as (1d) and (1e), with five and six syllables, respectively. With the six syllable string, the optimal candidate should include an initial degenerate foot. This suggests the prioritization of parsing, such that the relevant grammar will rank PARSE above RHTYPE $=$ A.

A disyllabic degenerate foot is (correctly) optimal

\begin{tabular}{|cc||c|c|}
\hline & $\sigma \sigma \sigma \sigma \sigma \sigma$ & PARSE & RHTYPE=A \\
\hline \hline a. & $\sigma \sigma(\sigma \sigma \sigma \sigma) \sigma$ & $* * ! *$ & \\
\hline b. & $(\sigma \sigma \sigma)(\sigma \sigma \sigma \sigma) \sigma$ & $*$ & $*$ \\
\hline
\end{tabular}

Recall however that (1d) shows the pattern $0(010)\langle 0\rangle$; the final syllable is extrametrical, the initial syllable is left unparsed, and the middle three form an amphibrach. Of course, the optimization of a form lacking a degenerate foot will fail with the grammar developed in (4), as seen in (5). 
A monosyllabic degenerate foot is (incorrectly) optimal

\begin{tabular}{|c|c|c|c|}
\hline & $\sigma \sigma \sigma \sigma \sigma$ & PARSE & RHTYPE=A \\
\hline \hline a. & $\sigma(\sigma \hat{\sigma} \sigma) \sigma$ & $* * !$ & \\
\hline b. & $(\sigma \dot{\sigma})(\sigma \hat{\sigma} \sigma) \sigma$ & $*$ & $*$ \\
\hline
\end{tabular}

Tableaux (4) and (5) bring out one of the central challenges for characterizing ternary rhythm, namely that there are two conceivable flavors of degenerate foot: binary and unary. In the Cayuvava patterns, and in analyses of other cases of ternary rhythm, degenerate feet are minimally binary; if the language is syllable counting, a degenerate foot is minimally disyllabic, if it is mora counting, the minimum is two morae.

Typological studies of stress patterns make it clear that there is variation regarding the footing of material smaller than a foot. For example, a language with a left-to-right trochaic stress pattern may or may not show stress on the final syllable of an odd-parity string. In OT, this can be modeled by changing the relative ranking of FOOTBINARITY and PARSE. When FoOTBINARITY is relatively high, a final lone syllable will be left unfooted. When PARSE is high, a final lone syllable will be footed.

We continue to assume for this discussion that the footing in (1) is the goal of an analysis, given foot boundary sensitive processes identified in other ternary rhythm languages, cf. Leer (1985); Rice (1992) inter alia. Under this assumption, the challenge of degenerate footing leaves us with a paradox. When a single syllable is left at the end of a parse, it remains unfooted, suggesting that PARSE does not compel ill-formed feet because it is lower ranked than the constraint compelling the correct foot type. When two syllables are left at the end of a parse, they are footed, suggesting that PARSE does compel ill-formed feet and should therefore be ranked above the foot type constraint. It's clear, then, that the relative ranking of PARSE and the 'don't parse' constraint (usually FOOTBINARITY, but in the present discussion RHTYPE=A) is not going to be enough to solve this problem. Candidates with monosyllabic feet will have to fail in another way, yet the generation of unary feet cannot be banned outright, since many languages with binary patterns show stress on stray monosyllables.

The problem with unary feet in ternary systems is not just that they are degenerate, but that they are too degenerate. Degenerate feet must be allowed, but only those which are minimally smaller than a full foot. By appealing to this generalization - that a degenerate foot is the size of a full foot minus one 
terminal - freedom of analysis could be restricted such that parses including ternary feet are prohibited from including unary ones. Alternatively, the generalization could be encoded in a constraint, preserving freedom of analysis and leaving the job of eliminating unary feet to CoN, albeit not as an effect of PARSE.

To the extent that all ternary systems prohibit unary ternary feet, a solution in CON would require a universal high ranking since the factorial typology of constraints would otherwise lead us incorrectly to expect variation on this point. Having to resort to a stipulated universal high ranking might itself be taken as a reason to read the restriction into GEN.

When (5b) is eliminated as a candidate by restricting GEN such that a parse with ternary feet cannot include unary feet, (5a) can be optimized. Of course, there are other candidates which must be considered in a more thorough discussion of the analysis of ternary stress, but our primary concern here has been to highlight the trade-offs between GEN and CON.

\subsection{Gen and CoN for amphibrachs}

Although a complete analysis using amphibrachs has not been offered above, we have seen enough to abandon the effort. This approach simply cannot be claimed to model any deep insights into the nature of the patterns. Feet with three syllables can be optimal, but this requires enhancing CoN with a constraint RHTYPE $=$ A which does nothing more than explicitly favor feet which are amphibrachs. And - as with the non-OT amphibrach analyses - the generalization about the size of degenerate feet is only available through stipulation, either in GEN or with a stipulatively highly ranked constraint in CON. Results achieved with ad hoc constraints rather than from constraint interaction are not particularly enlightening. So, although a descriptively correct analysis can be imagined by bringing the amphibrach into OT, the analysis is unsatisfying at several levels. ${ }^{4}$

As a final comment in this section, one might question how a constraint RHTYPE=I/T could be better formulated. Markedness constraints are usually formulated to punish ill-formed structures, yet this constraint is phrased positively. More fundamental, though, is that the constraint encodes at least two properties of feet, namely their size and their headedness. And, indeed, it probably encodes more, since $\mathrm{RHTYPE}=\mathrm{T}$, for example, is probably intended to punish not only iambs but also left-headed feet not present in the 


\section{Curt Rice}

Hayesian typology, (8), e.g. (HL) trochees. It would be preferably to derive the constellation of properties present in well-formed feet through the interaction of various constraints, such as FOOTBINARITY and perhaps alignment constraints positioning the head of a foot at either the right or left edge of the constituent; for related discussion see Eisner (1998). An enhancement of the constraint (family) to include the amphibrach, however, renders the alignment strategy is unavailable, since the correct position for the head of an amphibrach is exactly where misalignment is achieved; this is yet another reason to delay adoption of an analysis employing iterative amphibrachs.

\section{Weak local parsing in OT}

Questions about the division of labor between GEN and CON also arise when attempting to model ternary rhythm with binary feet, in the spirit of Hayes' (1985) weak local parsing. For example, if feet are universally maximally disyllabic, should GEN emit trisyllabic feet which are then ruled out by CON, or should GEN build maximally disyllabic feet, leaving to CON the responsibility for optimizing ternary rhythm without having to eliminate ternary feet? The present section presents a strategy for achieving ternary rhythm with binary feet in OT, drawing heavily on the analysis in Elenbaas and Kager (1999), and showing that this approach also makes crucial assumptions which may limit freedom of analysis.

\subsection{ALL-FoOT-L/R $\gg$ PARSE}

The footings seen in (3) will incur several violations of PARSE, e.g. four for the pattern in (i), $0(20) 0(20) 0(10) 0$. To make these parses optimal, they must be rewarded for their satisfaction of some constraint which dominates PARSE. The relevant constraint will be one which makes footing with fewer feet more harmonic than footing with more. The constraint invoked in the literature is ALL-FT-L/R, as proposed in McCarthy and Prince (1993a). This constraint examines each foot in a parse and awards violations for its distance from the left or right edge, using minimal violation to achieve the effect of directionality. When PARSE dominates ALL-FT-L/R, the effect will be a parse with iterative binary feet. But when ALL-FT-L/R dominates PARSE, fewer feet are favored. 
(6)

Minimize the number of feet

\begin{tabular}{|c|c||c|c|}
\hline & $\sigma \sigma \sigma \sigma$ & ALLFTR & PARSE \\
\hline \hline a. & $(\dot{\sigma} \sigma)(\dot{\sigma} \sigma)$ & $* ! *$ & \\
\hline b. & $\sigma \sigma(\hat{\sigma} \sigma)$ & & $* *$ \\
\hline
\end{tabular}

The two violations of ALL-FT-R in (6a) are associated with the leftmost foot in the string, which is two syllables away from the right edge of the word. When these syllables instead remain unfooted, as in (b), the alignment constraint is satisfied, but each of the unfooted syllables incurs a violation of PARSE.

The ranking in (6), however, goes too far in the pursuit of minimal parsing since it will always favor a parse with just one foot, even in longer strings. The weakly local parsed (7b) must defer to the severely underparsed (7c).

Optimize unipedal parses
\begin{tabular}{|c||c|c|}
\hline \multicolumn{1}{|c|}{$\sigma \sigma \sigma \sigma \sigma \sigma$} & ALLFTR & PARSE \\
\hline \hline a. $(\hat{\sigma} \sigma)(\hat{\sigma} \sigma)(\hat{\sigma} \sigma)$ & $* ! * * * *$ & \\
\hline b. $\sigma(\hat{\sigma} \sigma) \sigma(\hat{\sigma} \sigma)$ & $* ! * *$ & $* *$ \\
\hline c. $\sigma \sigma \sigma \sigma(\hat{\sigma} \sigma)$ & & $* * * *$ \\
\hline
\end{tabular}

So while the illustrated ranking of these two constraints does indeed contribute to favoring the nonexhaustive parses in (3) over exhaustive parsing, it is clear from (7) that the ranking ALL-FT-R over PARSE is not sufficient to generate ternary rhythm. Hence, the analysis must be enhanced by a constraint which stops short of PARSE, but which nonetheless limits a grammar's tolerance of unfooted material.

\subsection{Limiting unparsed syllables with * LAPSE}

Strings of unfooted syllables can be characterized as ill-formed because they represent unacceptably long spans lacking stress, creating a diseurythmic lapse in the string (Selkirk1984). Various *LAPSE constraints are to be found in the OT literature, e.g. Kager (1994), Green (1995), Gordon (2002), where the leading idea is that a string of more than two unstressed syllables is unacceptable.

Following Ishii (1966), an OT analysis of ternary rhythm can be based on 
the interaction of *LAPSE and ALL-FT-L/R. The latter compels relatively fewer feet and the former prevents long strings of unstressed syllables. Elenbaas and Kager's formulation of *LAPSE essentially says that an unstressed syllable must be adjacent to a stressed syllable or a word boundary. As we see in the following tableau, this formulation says nothing about footing, such that an unstressed syllable is licensed by a stressed one, independent of the position of foot boundaries, as with the medial unfooted syllables in (8b) and (8c).

Ternary rhythm with * LAPSE

\begin{tabular}{|c|c|c|c|c|c|}
\hline & & $\sigma \sigma \sigma \sigma \sigma \sigma$ & $*$ LAPSE & ALL-FT-R & PARSE \\
\hline & a. & $\left(\sigma \sigma^{\prime}\right)\left(\sigma \sigma^{\prime}\right)\left(\sigma \sigma^{\prime}\right)$ & & $* * * * * * !$ & \\
\hline & b. & $\left(\sigma \sigma^{\prime}\right) \sigma\left(\sigma \sigma^{\prime}\right) \sigma$ & & $* * * * ! *$ & $* *$ \\
\hline$\left[\begin{array}{ll}0 \\
0\end{array}\right.$ & c. & $\sigma(\sigma \hat{\sigma}) \sigma(\sigma \hat{\sigma})$ & & *** & ** \\
\hline & d. & $\sigma \sigma(\sigma \dot{\sigma})(\sigma \dot{\sigma})$ & $* !$ & $* *$ & $* *$ \\
\hline
\end{tabular}

Candidate (a) respects PARSE and thereby incurs the most violations of the higher ranked ALL-FT-R, four for the leftmost foot and two for the middle one. Among the candidates which violate PARSE twice, (c) is preferred to (b) under the pressure of ALL-FT-R. In both (b) and (c), *LAPSE is satisfied. The first syllable of candidate (c) does not violate *LAPSE even though it is not adjacent to a stressed syllable, because a word boundary also licenses an unstressed syllable. A * LAPSE violation, however, is awarded to candidate (d), where the second syllable is adjacent neither to a word boundary nor to a stressed syllable; with this fatal violation of *LAPSE, candidate (d)'s superior performance on ALL-FT-R is irrelevant.

\subsection{Elenbaas and Kager (1999)}

To apply this approach to the patterns in (3), we can consider the strategy advocated by Elenbaas and Kager (1999). I focus on their paper in part because of its explicitness regarding the division of labor. Specifially, Elenbaas and Kager set for themselves the goal of achieving a ternary parse through CON alone, modeling the patterns as an emergent effect of the interaction of independently motivated constraints.

We argue that these analyses [of ternary rhythm - CR] require no ternarity- 
inducing [emphasis theirs] mechanisms, such as ternary feet or special parsing modes. Instead, ternarity emerges by LICENSING, involving interactions of the anti-lapse constraint *LAPSE [...] with standard foot-alignment constraints [...]' (Elenbaas and Kager 1999: 274)

As we will see below, even the insightful analysis which Elenbaas and Kager present does require a crucial anti-ternarity limitation on freedom of analysis. But before making this point, the core properties of the analysis are presented. Their key insight for predicting the patterns in (3) is that both ALLFT-R and ALL-FT-L play a crucial role in the analysis. These constraints are crucially ranked, with ALL-FT-L dominating ALL-FT-R for the Cayuvava patterns. ALL-FT-L is crucial for selecting the optimal candidate when a string has the length $3 x$ while ALL-FT-R is crucial for strings with the length $3 x+2$ - the so-called double upbeat data. The optimal parse in the $3 x+1$ cases is selected by both ALL-FT-L and ALL-FT-R. Three tableaux are now presented, showing strings of four, five and six syllables, where the optimal candidates show the patterns from (3c-e).

Cayuvava 3x string a là Elenbaas \& Kager (1999)

\begin{tabular}{|r||c|c|c|c|}
\hline$\sigma \sigma \sigma \sigma \sigma \sigma$ & $*$ LAPSE & ALLFTL & ALLFTR & PARSE \\
\hline \hline a. $(\sigma \sigma \sigma) \sigma(\sigma \sigma) \sigma$ & & $* * *$ & $* * * * *$ & $* *$ \\
\hline b. $\sigma(\sigma \sigma \sigma)(\sigma \sigma) \sigma$ & & $* * * * !$ & $* * * *$ & $* *$ \\
\hline
\end{tabular}

All unstressed syllables in the candidates in (9) are adjacent either to stressed syllables or word boundaries, and *LAPSE is therefore unviolated. The first foot of candidate (a) is responsible for no violations of ALL-FT-L but for four of ALL-FT-R. The second foot of candidate (a) incurs three violations of ALL-FT-L and adds one more under ALL-FT-R. When the leftmost foot is not word initial, as in candidate (b), additional violations of ALL-FT-L are incurred but fewer of ALL-FT-R. Candidate (a) is therefore optimal. Because this correctly optimal candidate is inferior on ALL-FT-R, the tableau in (9) constitutes an argument for ranking ALL-FT-L above ALL-FT-R.

Tableau (10) differs from (9) insofar as the optimal candidate for strings having $3 x+1$ syllables will be best not only on ALL-FT-L but also on ALLFT-R.

The challenge in analyzing stress patterns from Cayuvava always arises with the $3 x+2$ strings. This is where Elenbaas and Kager's insight about the role of both ALL-FT-L/R constraints becomes clear. For these strings, 


\begin{tabular}{|cc||c|c|c|c|}
\hline & $\sigma \sigma \sigma \sigma$ & $*$ LAPSE & ALLFTL & ALLFTR & PARSE \\
\hline \hline a. & $\sigma(\sigma \sigma \sigma) \sigma$ & & $*$ & $*$ & $* *$ \\
\hline b. & $(\sigma \dot{\sigma} \sigma)(\sigma \dot{\sigma} \sigma)$ & & $* * !$ & $* *$ & \\
\hline
\end{tabular}

ALL-FT-L cannot be decisive because this constraint does not distinguish two initial syllables which are unfooted from two initial syllables which are footed, as seen in candidates (a) and (b) in (11). These candidates will be distinguished, however, by their performance on ALL-FT-R, which will favor the candidate in which those initial syllables are left unfooted. The lone foot in (a) is separated from the right edge by one syllable and therefore is awarded one asterisk under ALL-FT-R. The same violation is found in (b), but the initial foot there adds three more violations under this constraint, with the result that candidate (a) with the double upbeat is optimal.

Cayuvava $3 x+2$ string a là Elenbaas \& Kager (1999)

\begin{tabular}{|c|c|c|c|c|c|c|}
\hline & & $\sigma \sigma \sigma \sigma \sigma$ & *LAPSE & ALLFTL & ALLFTR & PARSE \\
\hline 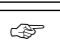 & $\overline{\mathrm{a} \text { a. }}$ & $\sigma \sigma(\hat{\sigma} \sigma) \sigma$ & & *** & * & $* * *$ \\
\hline & b. & $(\hat{\sigma} \sigma)(\hat{\sigma} \sigma) \sigma$ & & $* *$ & $* * ! * *$ & $*$ \\
\hline & c. & $(\hat{\sigma} \sigma) \sigma(\hat{\sigma} \sigma)$ & & $* * * !$ & **** & * \\
\hline
\end{tabular}

On the basis of (9), (10) and (11), it is clear that Elenbaas and Kager's approach successfully uses binary feet to get ternary rhythm. But because we are interested in the roles of GEN and CON, and because of freedom of analysis, we should also consider candidates with ternary feet. After all, an analysis which aspires to get ternary rhythm with binary feet as an emergent effect in CON must consider any output of GEN. In the next three tableaux, the optimal candidates from (9), (10) and (11) are shown to compare unfavorably with candidates making use of amphibrachs.

In each of these tableaux, the two candidates have stress on the correct syllables. The difference between them is that in each of the (a) candidates, the rightmost foot is a trochee preceded by an unfooted syllable, while in the (b) candidates, the rightmost foot is an amphibrach. The effect of this in each case is to move the leftmost boundary of the rightmost foot one syllable further to the left, such that the candidate with the amphibrach will be better by one when the performance of the two candidates on ALL-FT-L is evaluated. Since all the candidates satisfy *LAPSE, ALL-FT-L is decisive, and in each case, it favors the parse with an amphibrach. 
Cayuvava $3 x$ string allowing amphibrachs

\begin{tabular}{|c||c|c|c|c|}
\hline$\sigma \sigma \sigma \sigma \sigma \sigma$ & *LAPSE & ALLFTL & ALLFTR & PARSE \\
\hline \hline a. $(\sigma \sigma \sigma) \sigma(\sigma \sigma \sigma) \sigma$ & & $* * * !$ & $* * * * *$ & $* *$ \\
\hline b. $(\sigma \sigma \sigma)(\sigma \sigma \sigma \sigma) \sigma$ & & $* *$ & $* * * * *$ & $*$ \\
\hline
\end{tabular}

Cayuvava $3 x+1$ string allowing amphibrachs

\begin{tabular}{|cc||c|c|c|c|}
\hline & $\sigma \sigma \sigma \sigma$ & $*$ LAPSE & ALLFTL & ALLFTR & PARSE \\
\hline \hline a. & $\sigma(\sigma \sigma) \sigma$ & & $* !$ & $*$ & $* *$ \\
\hline b. & $(\sigma \sigma \sigma \sigma) \sigma$ & & & $*$ & $*$ \\
\hline
\end{tabular}

Cayuvava $3 x+2$ string allowing amphibrachs

\begin{tabular}{|rc||c|c|c|c|}
\hline & $\sigma \sigma \sigma \sigma \sigma$ & *LAPSE & ALLFTL & ALLFTR & PARSE \\
\hline \hline a. & $\sigma \sigma(\hat{\sigma} \sigma) \sigma$ & & $* * !$ & $*$ & $* * *$ \\
\hline b. & $\sigma(\sigma \sigma \sigma \sigma) \sigma$ & & $*$ & $*$ & $* *$ \\
\hline
\end{tabular}

In these tableaux, we see that the parses with amphibrachs harmonically bound the ones with trochees, such that no reranking can save the parses with binary feet. To save Elenbaas and Kager's analysis, the candidates with the amphibrachs must be eliminated in some other way. This, of course, can be accomplished either in GEN - by limiting freedom of analysis such that ternary feet are not produced - or in CON, perhaps with FOOTBINARITY. With the stated goal of deriving ternarity as an emergent effect, we should think that Elenbaas and Kager would propose eliminating these candidates with a constraint. And, clearly, when FOOTBINARITY is included in the grammar in a position dominating ALL-FT-L, the (b) candidates in (12), (13) and (14) will be eliminated. But as we see in the following section, an appeal to FOOTBINARITY masks other assumptions in this analysis.

\subsection{Binary vs. ternary systems}

The use of FOOTBINARITY to eliminate candidates with ternary feet is also important for Elenbaas and Kager's analysis of binary systems, and by looking at this analysis we will uncover important assumptions which impact their approach to ternarity. The basic difference between binary and ternary patterns for Elenbaas and Kager is in the relative rankings of ALL-FT-L/R and PARSE, as illustrated in $§ 5.1$ above. In binary systems, PARSE dominates ALL-FT-L/R, while in ternary systems, the ranking is reversed. FOOTBINA- 
RITY is explicitly stated as relevant for both systems, and it dominates the other two constraints. Highly ranked *LAPSE can be assumed for binary systems, although for our purposes it is irrelevant. The rankings are summarized in (15) and (16).

\section{Constraint ranking for binary rhythm}

$*$ LAPSE $\gg$ FOOTBINARITY $\gg$ PARSE $\gg$ ALL-FT-L/R

\section{Constraint ranking for ternary rhythm}

$*$ LAPSE $\gg$ FOOTBINARITY $\gg$ ALL-FT-L/R $\gg$ PARSE

The characterization of binary systems in (15) runs into trouble with systems which foot stray syllables, i.e. systems which have degenerate feet. In that situation, the ranking of FOOTBINARITY and PARSE must be reversed to make a candidate with a degenerate foot more harmonic than one with an unfooted syllable.

Degenerate feet as PARSE $\gg$ FOOTBINARITY

\begin{tabular}{|c|c|c|c|}
\hline & $\sigma \sigma \sigma$ & PARSE & FOOTBINARITY \\
\hline a. & $(\dot{\sigma} \sigma) \sigma$ & $* !$ & \\
\hline ix $b$. & $(\dot{\sigma} \sigma)(\dot{\sigma})$ & & $*$ \\
\hline
\end{tabular}

In identifying the candidates to be considered in (17), we assume only binary feet, which can either be proper or degenerate, in Hayes' terminology. But we must also consider a candidate with a ternary foot, lest we run afoul of the hypothesis of the richness of the base (cf. note 2). As shown in (18), such a candidate will perform just as well as the candidate with the degenerate foot, satisfying PARSE, and violating FOOTBINARITY once.

Equally optimal degenerate or ternary foot

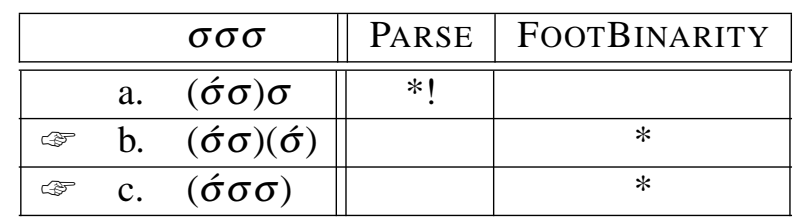

And, indeed, the situation is even worse, because ALL-FT-L and ALL-FT$\mathrm{R}$ are present in the grammar of binary systems, too; they're just relatively 
low ranked, as in (15). Add one of these to the tableau, and the parse with the amphibrach alone becomes optimal.

An optimal ternary foot

\begin{tabular}{|c|c|c|c|c|}
\hline & $\sigma \sigma \sigma$ & PARSE & FOOTBINARITY & ALLFTL \\
\hline & a. $\quad(\sigma \sigma \sigma) \sigma$ & $* !$ & & \\
\hline & b. $\quad(\dot{\sigma} \sigma)(\hat{\sigma})$ & & $*$ & $* ! *$ \\
\hline 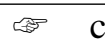 & c. $\quad(\sigma \sigma \sigma \sigma)$ & & * & \\
\hline
\end{tabular}

Again we face an analytical challenge which can be solved either in GEN or CON. If we solve this problem in GEN, we must restrict freedom of analysis to prohibit ternary feet. If we solve it in CoN, we need two different constraints to punish deviation from the binary ideal, e.g. $*[\sigma]_{\text {Foot }}$ and $*[\sigma \sigma \sigma]_{\text {Foot }}$. In languages with no degnerate feet, $*[\sigma]_{\text {Foot }}$ asserts itself as highly ranked. But when PARSE is ranked between these two constraints, with $*[\sigma \sigma \sigma]_{\text {Foot }}$ highest, ternary feet will be ruled out while unary feet will be allowed.

Eliminating the ternary foot with $*[\sigma \sigma \sigma]_{F t}$

\begin{tabular}{|c|c|c|c|c|c|}
\hline & $\sigma \sigma \sigma$ & $*[\sigma \sigma \sigma]_{F t}$ & PARSE & $*[\sigma]_{F t}$ & ALLFTL \\
\hline & a. $(\hat{\sigma} \sigma) \sigma$ & & $* !$ & & \\
\hline 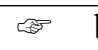 & b. $\quad(\dot{\sigma} \sigma)(\dot{\sigma})$ & & & $*$ & $* *$ \\
\hline & c. $(\hat{\sigma} \sigma \sigma)$ & $* !$ & & * & \\
\hline
\end{tabular}

Freedom of analysis opens for any amount of structure, such that an analysis of binary rhythm must consider candidates with ternary feet. The ranking necessary to analyze a language with unary feet at the end of a string turns out to be a ranking under which a string-final ternary foot is more harmonic than a string-final sequence of a binary foot followed by a unary one. Because FOOTBINARITY - or at least $*[\sigma]_{F t}$ - must be relatively low ranked when unary feet are allowed, an optimal string final ternary foot has to be ruled out either by prohibiting ternary feet in GEN through a restriction on freedom of analysis, or with a highly ranked constraint specifically devoted to ruling out ternary feet. 


\section{Curt Rice}

\subsection{Gen and CON in a model of ternary rhythm with binary feet}

The solution used for analyzing binary systems will of course also be on the scene for the analysis of ternary rhythm. We noted above that highly ranked FoOTBINARITY might do the job necessary in (12), (13) and (14) by punishing the candidates with an amphibrach in their parse. But, in fact, Elenbaas and Kager have no need to refer to FOOTBINARITY in this context. The reason for this is that their analysis of binary systems - in particular those which allow degenerate feet - requires either a restriction on freedom of analysis blocking trisyllabic feet or a universally highly ranked constraint such as $*[\sigma \sigma \sigma]_{F t}$. Either way, FOOTBINARITY is supplemented by restrictions on GEN or else replaced by more specific constraints in CON. For this reason, I suggest that the elimination of the ternary feet in those tableaux is not really accomplished by FOOTBINARITY, and the appeal to that constraint has the effect of masking a more complicated situation that includes ternary-specific mechanisms.

Given the arguments made here, one might ask whether Elenbaas and Kager achieve their stated goal of modeling ternary stress without any 'ternaryinducing mechanisms'. Since nothing in their analysis induces ternary feet, we can concede that they have achieved their stated goal.

But analyses in OT don't need to induce structure; structure appears without motivation according to the hypotheses of the richness of the base and freedom of analysis. Because of these methodological assumptions in classical OT, the task of an analysis is not to avoid inducing a particular structure, but rather to provide a principled strategy for eliminating an unwanted structure, even one which is proposed to be universally absent.

If the correct analysis of ternary rhythm involves non-exhaustive parsing with binary feet, then the goal of an analysis must be to achieve such a parsing with no ternary-specific mechanisms in a way which nonetheless prefers binary feet to ternary ones; in short, parses with binary feet must harmonically bound parses with ternary ones. Ternary rhythm with binary feet must be achieved through the interaction of violable constraints, not through a ternaryspecific stipulation in GEN or with a ternary-specific constraint stipulated as universally highly ranked. This is the currently unmet challenge for future attempts to develop emergent analyses of ternary rhythm. 


\section{Conclusion}

The presentation in this paper aims to identify situations in which we are forced to look at GEN and to entertain restrictions on freedom of analysis as a strategy worthy of consideration alongside a possible solution in CON. The specific case under review involves the modeling of ternary rhythm, and the issues which arise there may be somewhat more subtle than definitional restrictions on GEN such as prohibiting crossing association lines. To say that GEN produces maximally disyllabic feet is no less stipulative than the claim that GEN produces maximally trisyllabic feet; both of these restrictions, stated in this straightforward way, are stipulations without any principled foundation.

The universal absence of ternary feet may be a hypothesis worth pursuing and it may be a consequence of a more insightful restriction on GEN - for example, specific properties of constituent structure. To move in the direction of a deeper understanding of freedom of analysis and its implications for modeling grammar and typology, we have to study GEN. We have to look at cases in which different assumptions about GEN have an impact on the details of the analysis being pursued, and then consider the relative merits of these various assumptions. Assumed restrictions on GEN can surely be found in most OT analyses; one never finds, for example, a candidate set for an analysis of stress including candidates which represent prominence with the grid alone (Prince 1983) alongside candidates which use footing, leaving it to CON to distinguish these approaches. Neither does one find analyses of syllable-based phenomena in which structures with 'every conceivable array of affiliations and empty and filled nodes' are actually considered (op.cit.). Freedom of analysis is rarely assumed to be truly free. The assumptions hidden in typical analyses must be made explicit.

By questioning GEN and asserting that research on this aspect of the theory is wanting, I take a different view than the one advocated by McCarthy (2002: 8), where we read that '[i]n phonology, there is a rough consensus about the properties of GEN.' It's difficult to imagine the basis for this claim. Certainly, one cannot reach that conclusion by reviewing the practice seen in most OT analyses, where the assumptions or properties of GEN are rarely mentioned.

But beyond practical matters, if GEN is the repository of truly universal formal structures, then McCarthy's claim boils down to an assertion that there is a consensus about such structures. Yet in fundamental areas such 


\section{Curt Rice}

as the inventory of features, feature geometry, the structure of the syllable, or the inventory of metrical feet, the contemporary literature offers little basis for inferring consensus. OT doesn't require the abandonment of research on structure but it does require reconceptualization of how results related to phonological structure should be modeled. Consensus on these matters is a respectable objective, but it does not currently exist.

The quest for results emerging from the interaction of independently motivated constraints should be pursued in explicit awareness that the tools and alphabet available to UG might be limited, and that these limitations are relevant for analyses and loaded with the potential for actual insights, not least of all about the properties of linguistic structures and thereby the properties of the candidates submitted to Eval. The nature of such limitations is a legitimate focus of research, and it is one which we can hope will now assume its rightful place alongside the study of constraints and their interaction.

\section{Notes}

*. This paper has emerged from presentations at the Workshop on Freedom of Analysis in Troms $\varnothing$, a University College London colloquium, the Variation and Stability in Grammar workshop in Nijmegen and the Fourth North American Phonology Conference in Montreal; I thank the members of those audiences for their valuable input. I'm also grateful to my fellow phonologists at the Center for Advanced Study in Theoretical Linguistics (CASTL) in Troms $\emptyset$ - particularly Sylvia Blaho, Patrik Bye, Martin Krämer, Ove Lorentz, Bruce Morén, and Christian Uffmann - for discussion of the ideas presented here, and for the input of two anonymous reviewers.

1. A third strategy whereby such structures are simply left unconsidered by the grammar is precluded by Prince and Smolensky's (1993) hypothesis of the richness of the base. This hypothesis, or methodology, requires that we achieve surface results through formalized generalizations about surface structures, rather than by restricting potential inputs.

2. In the discussion that follows, I consider only an OT model using harmonic parallelism and not one using harmonic serialism. These two different strategies for implementing OT differ rather fundamentally in their conception of freedom of analysis. As I noted in Rice (2005: 6), 'the pursuit of a restricted theory of GEN might be facilitated by a closer examination of the serial approach to OT and a fitting version of freedom of analysis'. For related discussion, see McCarthy (2007).

3. Relevant references include Leer (1985); Levin (1985); Halle and Vergnaud (1987); Everett (1988); Levin (1988); Rice (1988); Halle (1990); Hammond (1990); Dresher and Lahiri (1991); Hewitt (1992); Rice (1992, 1993); Kager (1994); Kenstowicz (1994); Green (1995); Green and Kenstowicz (1995); Hammond (1995); Hayes (1995); Ishii (1996); Berry (1998); van de Vijver (1998); Blevins and Harrison (1999); Elenbaas (1999); Elenbaas and Kager (1999); van der Hulst (1999); Hyde (2001); Gordon (2002); Hyde (2002); Rifkin (2003); Houghton (2006).

4. Another option for eliminating a unary foot as a candidate degenerate ternary foot is to 
build on Rice's (1992) proposal that ternary feet have internal structure, and that two of the three syllables in the foot form a head. The restriction which one would then propose for GEN is that a constituent consists minimally of a head, cf. relevant discussion in Dresher and van der Hulst (1998). In the case of a trisyllabic foot with a disyllabic head, this has the effect of ruling out monosyllabic feet, correctly capturing the generalization about the degenerate feet in the Cayuvava patterns. This is not available in the amphibrachic approach, where the matter requires explicit stipulation. Space restrictions preclude further discussion of this approach here.

\title{
References
}

\author{
Berry, Lynn \\ 1998 Alignment and adjacency in optimality theory: Evidence from Warlpiri and \\ Arrernte. Ph. D. diss., University of Sydney. ROA 271. \\ Blevins, Juliette, and Sheldon P. Harrison \\ 1999 Trimoraic feet in Gilbertese. Oceanic Linguistics 38:203-230. \\ Dresher, B. Elan, and Harry van der Hulst \\ 1998 Head-dependent asymmetries in phonology: Complexity and visibility. Phonol- \\ ogy 15:317-352.
}

Dresher, B. Elan, and Aditi Lahiri

1991 The Germanic foot: Metrical coherence in Old English. Linguistic Inquiry 22:251-286.

Eisner, Jason

1998 FOOTFORM decomposed: Sing primitive constraints in OT. In MIT working papers in linguistics, Benjamin Bruening (ed.), volume 31, 115-143. MIT.

Elenbaas, Nine

1999 A unified account of binary and ternary stress: Considerations from Sentani and Finnish. Ph. D. diss., Utrecht University.

Elenbaas, Nine, and René Kager

1999 Ternary rhythm and the *LAPSE constraint. Phonology 16:273-313.

Everett, Daniel L

1988 On metrical constituent structure in Piraha phonology. Natural Language and

Linguistic Theory 6:207-246.

Gordon, Matthew

2002 A factorial typology of quantity-insensitive stress. Natural Language and Linguistic Theory 20:491-552.

Green, Thomas

1995 The stress window in Piraha: A reanalysis of rhythm in optimality theory. ROA 45.

Green, Thomas, and Michael Kenstowicz 1995 The lapse constraint. ROA 101.

Halle, Morris

$1990 \quad$ Respecting metrical structure. Natural Language and Linguistic Theory 8:149176. 


\section{Curt Rice}

Halle, Morris, and Jean-Roger Vergnaud 1987 An Essay On Stress. Cambridge, Mass.: MIT Press.

Hammond, Michael

1990 Deriving ternarity. Ms., University of Arizona, Tucson.

1995 Metrical phonology. Annual review of anthropology 24:313-42.

Hayes, Bruce

1995 Metrical Stress Theory: Principles And Case Studies. Chicago: Chicago University Press.

Hewitt, Mark

1992 Vertical maximization and metrical theory. Ph. D. diss., Brandeis University.

Houghton, Paula

2006 Ternary stress. ROA 836.

van der Hulst, Harry

1999 Word accent. In Word Prosodic Systems in the Languages of Europe, Harry van der Hulst (ed.), 3-96. Berlin: Mouton de Gruyter.

Hyde, Brett

2001 Metrical and prosodic structure in optimality theory. Ph. D. diss., Rutgers, The State University of New Jersey. ROA 476.

2002 A restrictive theory of metrical stress. Phonology 19:313-359.

Ishii, Toru

1996 An optimality theoretic approach to ternary stress systems. In Proceedings of the South Western Optimality Theory Workshop (SWOT II). UCI Working papers in linguistics, volume 2, 95-111. Irvine, CA: University of California, Irvine.

Kager, René

1994 Ternary rhythm in alignment theory. Ms., Utrecht University, ROA 35.

Kenstowicz, Michael

$1994 \quad$ Phonology in Generative Grammar. Oxford: Blackwell.

Key, Harold H.

1961 Phonotactics of Cayuvava. International Journal of American Linguistics 27:143-

150 .

1967 Morphology of Cayuvava. Janua Linguarum, Series Practica 50. The Hague: Mouton.

Leer, Jeff

1985 Prosody in Alutiiq. In Yupik Eskimo Prosody Systems: Descriptive And Com-

parative Studies, Michael Krauss (ed.), 77-133. Fairbanks: University of Alaska.

Levin, Juliette

1985 Evidence for ternary feet and implications for a metrical theory of stress rules.

Ms., University of Texas at Austin.

1988 Generating ternary feet. Texas Linguistic Forum 29:97-113.

McCarthy, John J.

2002 A Thematic Guide to Optimality Theory. Cambridge: Cambridge University Press.

2007 Restraint of analysis. This volume. Also published in Wondering at the Natural Fecundity of Things: Essays in Honor of Alan Prince, http://repositories.cdlib.org/lrc/prince/10. 
McCarthy, John J., and Alan Prince

1993a Generalized alignment. In Yearbook of Morphology, ed. Geert Booij and Jaap van Marle, 79-153. Dordrecht: Kluwer.

1993b Prosodic morphology I. Constraint interaction and satisfaction. Ms., University of Massachusetts, Amherst and Brandeis University; ROA 482.

Prince, Alan

$1983 \quad$ Relating to the grid. Linguistic Inquiry 14:19-100.

Prince, Alan S., and Paul Smolensky

1993 Optimality theory. Constraint interaction in generative grammar. Technical Report \#2, Rutgers University Center for Cognitive Science. ROA 537; Published in 2004 by Blackwell Publishers).

Rice, Curt

1988 Stress assignment in the Chugach dialect of Alutiiq. In CLS 24: Papers from the $24^{\text {th }}$ Annual Regional Meeting of the Chicago Linguistic Society, Lynn MacLeod, Gary Larson, and Diane Brentari (eds.), 304-315. Chicago, IL: Chicago Linguistic Society.

1992 Binarity and ternarity in metrical theory: Parametric extensions. Ph. D. diss., University of Texas at Austin. Available at http://www.hum.uit.no/a/rice.

1993 A note on ternary stress in Sentani. In University of Trondheim Working Papers in Linguistics, 67-71. Trondheim: Department of Linguistics, University of Trondheim.

2005 Freedom $\nRightarrow$ anarchy. Presented at Workshop on Freedom of Analysis, September 1-2, 2005, Troms $\emptyset$.

Rifkin, Jay I.

2003 Ternarity is prosodic word binarity. In The Phonological Spectrum, Volume II: Suprasegmental Structure, Jeroen van de Weijer, Vincent J. van Heuven, Selkirk, Elisabeth and Harry van der Hulst (eds.), 127-150. Amsterdam: John Benjamins.

1984 Phonology and Syntax: The Relation Between Sound And Structure. Cambridge, Mass.: MIT Press.

van de Vijver, Ruben

1998 The iambic issue: Iambs as a result of constraint interaction. HIL Dissertations 37. Holland Institute of Generative Linguistics. 